\title{
Methotrexate-related lymphoproliferative disorder of the lumbar spine origin presenting with severe low-back pain: case report
}

\author{
Naoya Kikuchi, MD, ${ }^{1}$ Masafumi Uesugi, MD, PhD, ${ }^{1}$ Masao Koda, MD, PhD, ${ }^{2}$ Tomoaki Shimizu, MD, ${ }^{1}$ \\ Kohei Murakami, MD, ${ }^{1}$ Mamoru Kono, MD, ${ }^{1}$ Haruka Tanaka, MD, ${ }^{1}$ and Masashi Yamazaki, MD, PhD ${ }^{2}$ \\ 1Department of Orthopaedic Surgery, Ibaraki Seinan Medical Center Hospital, Sashima, Ibaraki; and 2Department of Orthopaedic \\ Surgery, Faculty of Medicine, University of Tsukuba, Ibaraki, Japan
}

\begin{abstract}
The use of methotrexate (MTX) to treat rheumatoid arthritis (RA) is increasing. Recently, MTX-associated lymphoproliferative disorder (MTX-LPD) has been frequently reported as lymphoma occurring during MTX therapy. The authors report their experience with a relatively rare case of MTX-LPD presenting in the lumbar spine. The patient, a 73-year-old woman who experienced low-back pain while receiving MTX therapy for RA, was suspected of having developed MTXLPD based on her medical history, images of the L1 vertebra, and transpedicular biopsy results. One week after discontinuing MTX, the patient's low-back pain reportedly improved. The woman was diagnosed with MTX-LPD based on histopathological findings. MTX discontinuation alone coincided with spontaneous tumor regression. Because MTX-LPD can occur in tissues other than lymph nodes, such as in bones and joints, it is a disease that should be considered when diagnosing spinal tumors in patients receiving MTX therapy.

https://thejns.org/doi/abs/10.3171/2018.4.SPINE1860
\end{abstract}

KEYWORDS rheumatoid arthritis; methotrexate; lymphoproliferative disorder; lumbar spine; oncology

$\mathrm{M}$ ETHOTREXATE (MTX) is a highly effective treatment recommended for rheumatoid arthritis (RA) based on guidelines. In Japan, the estimated prevalence of RA was 1.24 million and MTX was prescribed to 329,000 individuals. ${ }^{14}$ However, serious adverse effects are widely known to be associated with MTX therapy, including liver disorders, interstitial pneumonia, and myelosuppression, among others. Of the various adverse effects associated with MTX therapy, lymphoma was the first to be reported in a patient with RA in $1991 .{ }^{1}$ Since that time, lymphoma has been attracting attention as an MTX-associated lymphoproliferative disorder (MTXLPD). Here, we report a case of MTX-LPD in the lumbar spine of a patient with RA who was treated with MTX.

\section{Case Report}

\section{History and Examination}

A 73-year-old woman presented with low-back pain in the absence of any triggers, such as trauma. The patient visited our department because her low-back pain was severe, resulting in reduced mobility. The patient was diag- nosed with RA at 69 years of age and was immediately started on a regimen of 6-10 $\mathrm{mg} / \mathrm{wk}$ MTX after being diagnosed. Her approximate cumulative dose of MTX was $1800 \mathrm{mg}$. Routine follow-up visits were performed at our hospital's rheumatology department.

On admission, the patient reported severe pain in the upper lumbar spine. No neurological abnormalities of the lower limbs were observed. A laboratory investigation revealed a normal lactate dehydrogenase level (211 IU/L), a slightly elevated C-reactive protein level $(2.09 \mathrm{mg} / \mathrm{ml})$, apparent elevation in the soluble interleukin-2 receptor value (3220 U/ml), and an increased Epstein-Barr virus (EBV) immunoglobulin $\mathrm{G}$ antibody titer.

MRI revealed low signal intensity in the $L 1$ vertebral body on both T1- and T2-weighted images, as well as a soft-tissue mass compressing the dural sac (Fig. 1). Contrast-enhanced CT showed swollen cervical and mediastinal lymph nodes on the left.

\section{Pathological Findings}

We performed a transpedicular biopsy of the L1 vertebral body to rule out a suspected spinal tumor based on

ABBREVIATIONS EBV = Epstein-Barr virus; $L P D=$ lymphoproliferative disorder; MTX = methotrexate; $M T X-L P D=M T X$-associated LPD; RA = rheumatoid arthritis. SUBMITTED January 15, 2018. ACCEPTED April 19, 2018.

INCLUDE WHEN CITING Published online August 31, 2018; DOI: 10.3171/2018.4.SPINE1860. 


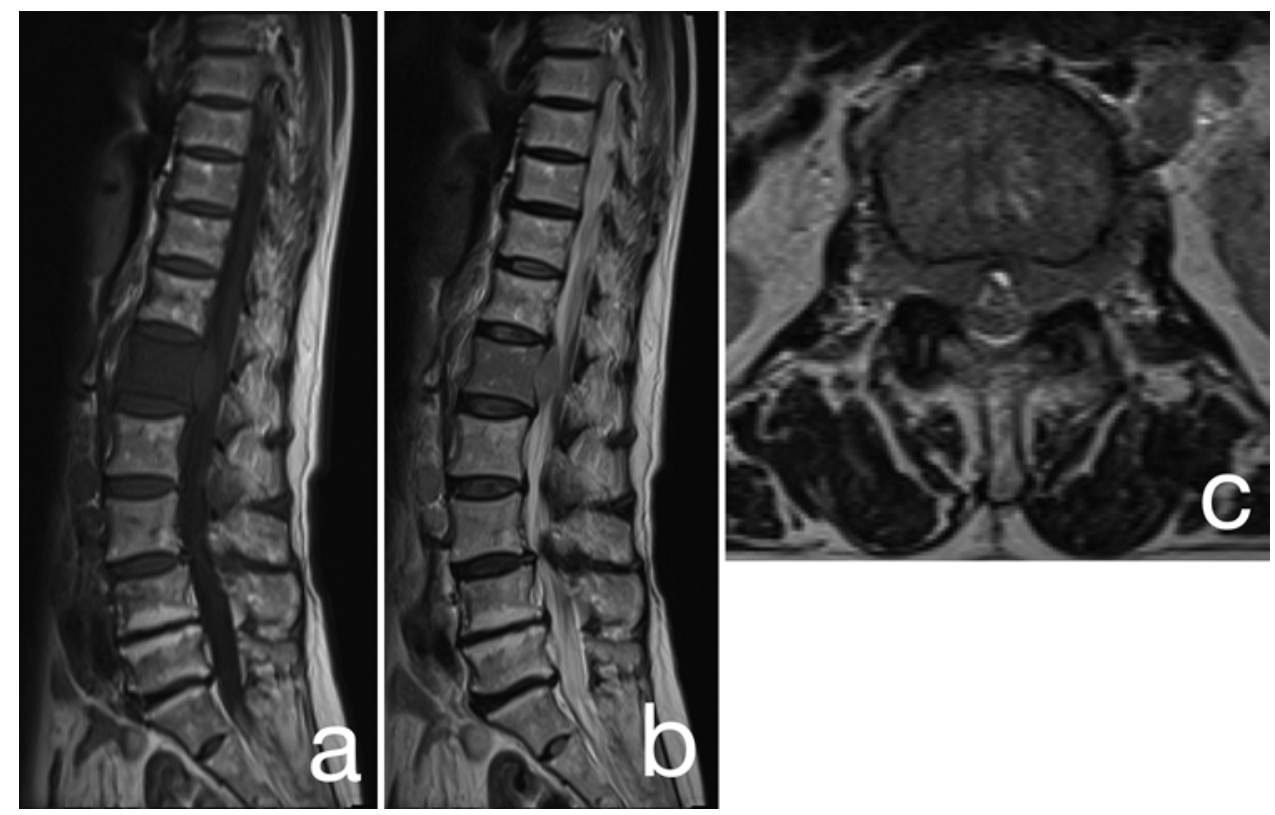

FIG. 1. Sagittal T1- (a) and T2- (b) weighted MR images and axial T2-weighted MR image (c) obtained at the L1 vertebra at admission, showing an epidural mass with low signal intensity.

the imaging findings. Examination revealed pathological trabecular destruction and diffuse infiltration of lymphoid cells in the inter-trabecular spaces with fibrilization in the background (Fig. 2a). Epstein-Barr encoding region in situ hybridization indicated EBV positivity (Fig. 2b). Immunohistochemical examination showed large atypical CD30positive cells and a scattered distribution of PAX5-positive cells (Fig. 2c and d), suggesting a diagnosis of Hodgkin's lymphoma-like LPD.

\section{Clinical Outcome}

MTX therapy was discontinued based on a working diagnosis of MTX-LPD. The patient regained the ability to walk unassisted 8 days after the discontinuation of MTX. Soluble IL-2 receptor levels decreased to $565 \mathrm{U} /$ $\mathrm{ml} 1$ month after MTX discontinuation, and a contrastenhanced CT scan revealed regression of the swollen lymph nodes. Follow-up MRI showed attenuation of both the epidural mass compressing the dural sac and the signal changes in the L1 vertebral body. Residual signal changes and the collapse of the superior lumbar endplate were observed, suggesting a vertebral fracture (Fig. 3). Radiography and MRI 6 months after MTX discontinuation showed no signs of MTX-LPD recurrence.

\section{Discussion}

MTX-LPD is a lymphoproliferative disorder that can occur in patients receiving MTX therapy for diseases such as RA; it is classified as the "other iatrogenic subgroup of immunodeficiency-associated lymphoproliferative disorders" according to the 2008 WHO Classification of Tumours of Haematopoietic and Lymphoid Tissues. ${ }^{12}$ MTX-LPD is a generic term referring to all the LPDs that develop in patients on MTX therapy and can include common LPD as well as LPD accompanying RA, both of which are difficult to differentiate.

The symptoms of MTX-LPD are B symptoms similar to those in normal lymphoma (high fever, weight loss, and night sweats). MTX-LPD is not characterized by specific histology, and the reported proportions of histology consist of $52 \%$ diffuse large B-cell lymphoma and $8.9 \%$ Hodgkin's lymphoma. ${ }^{4} \mathrm{EBV}$ is said to be associated with MTX-LPD, and 30\%-50\% of patients affected by this disease have been reported to be EBV positive. ${ }^{3,8,11} \mathrm{Com}$ pared with EBV-negative cases, EBV-positive MTX-LPD cases often undergo spontaneous regression by only discontinuing MTX, ${ }^{3}$ suggesting that the tumor cells proliferate due to MTX-induced suppression of cellular immunity. ${ }^{9}$ MTX-LPD commonly develops $40 \%-50 \%$ of the time as extralymphatic lesions mainly in the gastrointestinal tract, skin, liver, and lungs. ${ }^{3,11}$ MTX-LPD can also occur in the musculoskeletal system, and bone marrow infiltration accounts for the pathology in approximately $3 \%$ of all MTX-LPD patients. ${ }^{6}$ Cases of MTX-LPD developing in the metacarpal-phalangeal joints ${ }^{7}$ and the elbow joint ${ }^{2}$ have been previously reported. For the first time, we report a rare case of MTX-LPD originating from the lumbar spine. However, the absence of similar previous reports at this time should not preclude MTX-LPD from being considered in the differential diagnosis for spinal tumors in patients receiving MTX therapy. Our finding confirms that MTX-LPD can develop in the spine similar to other lymphoproliferative disorders.

Reportedly, $60 \%$ of MTX-LPD cases spontaneously regress when MTX is discontinued, suggesting that tumor cells may proliferate in response to MTX-induced immunosuppression. ${ }^{9}$ Reports have indicated that spontaneous regression can easily be achieved in cases of EBV-positive, nondiffuse large B-cell lymphoma com- 


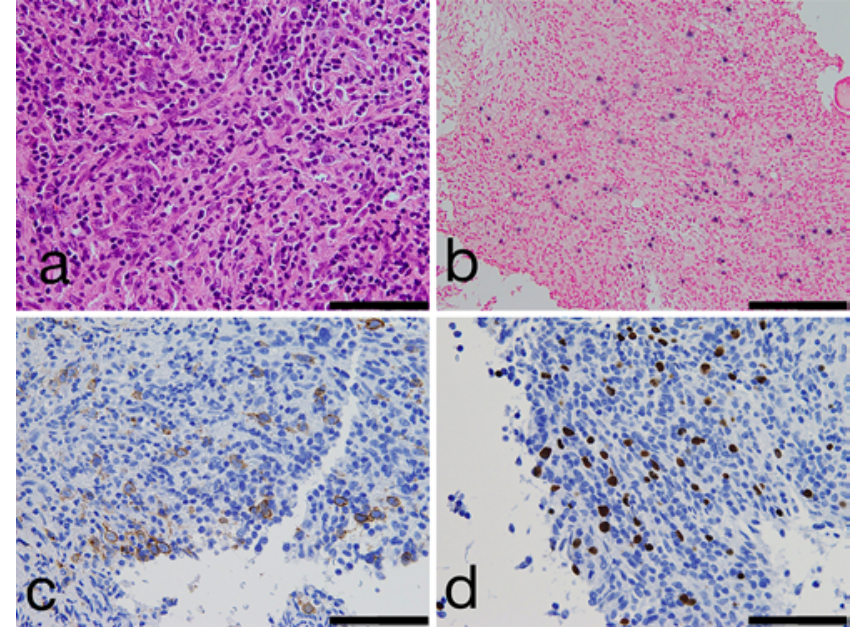

FIG. 2. a: Histological findings on the pedicle of the arch of the L1 vertebra showing trabecular destruction and diffuse infiltration of lymphoid cells in the inter-trabecular spaces with fibrilization. H \& E. b-d: Findings from immunohistochemical analysis showing positive staining for the Epstein-Barr encoding region via in situ hybridization (b), CD30 (c), and PAX5 (d). Bar $=40 \mu \mathrm{m}$. Figure is available in color online only.

pared with other types of LPD. ${ }^{4}$ Therefore, biopsy and EBV testing, as well as MTX discontinuation, are required when MTX-LPD is the working diagnosis. The optimal duration for observation remains unclear at this time in the presence of conflicting reports describing the different durations required for spontaneous regression of MTX-LPD after the discontinuation of MTX therapy. Most previous reports have indicated that the spontaneous regression of MTX-LPD takes approximately 2 weeks from the time of MTX discontinuation, although one report described a case in which spontaneous regression took 8 weeks. ${ }^{5}$ It is imperative that an optimal duration for the observation of spontaneous regression of MTX-LPD after MTX discontinuation be explored and established in order to not delay chemotherapy as the next line of defense.

Even if the spontaneous regression was achieved by MTX discontinuation, a previous study reported that during a mean follow-up of 34 months, among 8 patients who were treated by MTX discontinuation alone, 2 had a relapse 12 to 14 months later. ${ }^{10}$ According to the result of this report, follow-up is necessary for at least 2 years, although it is impossible to clearly state the optimal followup period at present.

Biopsy is an important tool to determine the precise treatment strategy under appropriate diagnosis in patients with spinal tumors. Operative treatment would be considered on the basis of the indications for neurological impairment and/or local instability. In the spinal tumor cases without neurological compromise and segmental instability, needle biopsy rather than surgical biopsy appears to be more suitable as an initial treatment option because of its fewer complications and lower cost in comparison with those of surgical biopsy. ${ }^{13}$ In the current case, we decided to perform a needle biopsy rather than surgery, because no neurological symptoms and/or local instability were
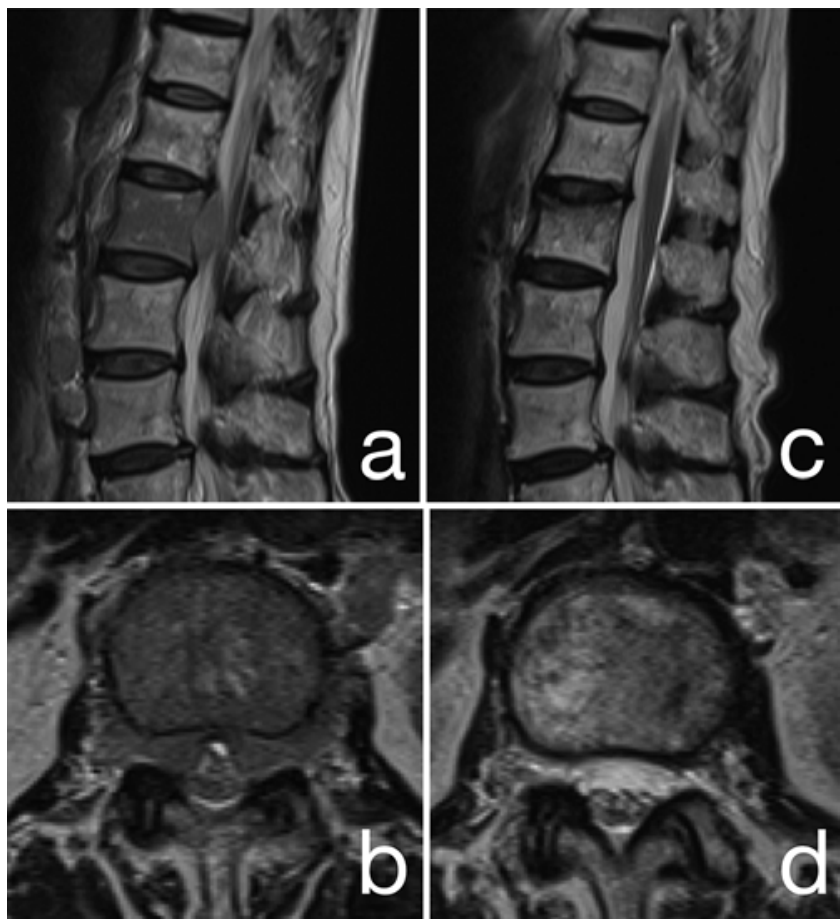

FIG. 3. Sagittal (a) and axial (b) T2-weighted MR images obtained at L1 on admission, and sagittal (c) and axial (d) T2-weighted MR images obtained at L1 3 months after MTX discontinuation. Note the comparative attenuation of both the epidural mass compressing the dural sac and signal changes in the vertebral body after 3 months.

found, even though a large vertebral tumor with epidural extension was observed.

The patient in the present case was diagnosed with MTX-LPD when she presented with low-back pain. The disease course was typical for a patient with EBV-positive, nondiffuse large B-cell lymphoma that achieved spontaneous regression by MTX discontinuation alone, as reported previously.

The patient should be followed up and rigorously observed, even when there is no sign of recurrence at a follow-up visit as late as 6 months after an apparent spontaneous regression of MTX-LPD.

\section{Conclusions}

We report the case of an MTX-LPD originating in the lumbar spine. Although spontaneous regression was achieved by MTX discontinuation alone, the patient requires continuous and rigorous follow-up at 6-month intervals. MTX-LPD should be considered as one of the differential diagnoses of spinal tumors in patients receiving MTX therapy.

\section{Acknowledgments}

We thank to Hidekazu Nishikii, Hiroshi Ebe, and Yoko Yano for making a diagnosis.

\section{References}

1. Ellman MH, Hurwitz H, Thomas C, Kozloff M: Lymphoma 
developing in a patient with rheumatoid arthritis taking low dose weekly methotrexate. J Rheumatol 18:1741-1743, 1991

2. Hatano T, Ohishi M, Yoshimoto G, Yamauchi M, Maekawa A, Yamamoto H, et al: Methotrexate-related lymphoproliferative disorder presenting with severe swelling of the elbow joint: a case report. JBJS Case Connect 7:e65, 2017

3. Hoshida Y, Xu JX, Fujita S, Nakamichi I, Ikeda J, Tomita Y, et al: Lymphoproliferative disorders in rheumatoid arthritis: clinicopathological analysis of 76 cases in relation to methotrexate medication. J Rheumatol 34:322-331, 2007

4. Ichikawa A, Arakawa F, Kiyasu J, Sato K, Miyoshi H, Niino $\mathrm{D}$, et al: Methotrexate/iatrogenic lymphoproliferative disorders in rheumatoid arthritis: histology, Epstein-Barr virus, and clonality are important predictors of disease progression and regression. Eur J Haematol 91:20-28, 2013

5. Inui Y, Matsuoka H, Yakushijin K, Okamura A, Shimada T, Yano S, et al: Methotrexate-associated lymphoproliferative disorders: management by watchful waiting and observation of early lymphocyte recovery after methotrexate withdrawal. Leuk Lymphoma 56:3045-3051, 2015

6. Kameda T, Dobashi H, Miyatake N, Inoo M, Onishi I, Kurata $\mathrm{N}$, et al: Association of higher methotrexate dose with lymphoproliferative disease onset in rheumatoid arthritis patients. Arthritis Care Res (Hoboken) 66:1302-1309, 2014

7. Kennedy JW, Wong LK, Kalantarian B, Turner L, Hayes $\mathrm{CW}$ : An unusual presentation of methotrexate-induced B-cell lymphoma of the metacarpophalangeal joint: a case report and literature review. J Hand Surg Am 31:1193-1196, 2006

8. Kikuchi K, Miyazaki Y, Tanaka A, Shigematu H, Kojima M, Sakashita H, et al: Methotrexate-related Epstein-Barr Virus (EBV)-associated lymphoproliferative disorder-so-called "Hodgkin-like lesion"-of the oral cavity in a patient with rheumatoid arthritis. Head Neck Pathol 4:305-311, 2010

9. Kremer JM: Is methotrexate oncogenic in patients with rheumatoid arthritis? Semin Arthritis Rheum 26:785-787, 1997

10. Mariette X, Cazals-Hatem D, Warszawki J, Liote F, Balandraud N, Sibilia J: Lymphomas in rheumatoid arthritis patients treated with methotrexate: a 3-year prospective study in France. Blood 99:3909-3915, 2002
11. Salloum E, Cooper DL, Howe G, Lacy J, Tallini G, Crouch $\mathrm{J}$, et al: Spontaneous regression of lymphoproliferative disorders in patients treated with methotrexate for rheumatoid arthritis and other rheumatic diseases. J Clin Oncol 14:1943-1949, 1996

12. Swerdlow SH, Campo E, Harris NL, Jaffe ES, Pileri SA, Stein H, et al: WHO Classification of Tumours of Haematopoietic and Lymphoid Tissues. Lyon, France: IARC Press, 2008

13. Welker JA, Henshaw RM, Jelinek J, Shmookler BM, Malawer MM: The percutaneous needle biopsy is safe and recommended in the diagnosis of musculoskeletal masses. Cancer 89:2677-2686, 2000

14. Yamanaka H, Sugiyama N, Inoue E, Taniguchi A, Momohara $\mathrm{S}$ : Estimates of the prevalence of and current treatment practices for rheumatoid arthritis in Japan using reimbursement data from health insurance societies and the IORRA cohort (I). Mod Rheumatol 24:33-40, 2014

\section{Disclosures}

The authors report no conflict of interest concerning the materials or methods used in this study or the findings specified in this paper.

\section{Author Contributions}

Conception and design: Kikuchi. Acquisition of data: Kikuchi. Drafting the article: Kikuchi. Critically revising the article: Kikuchi, Uesugi, Koda. Reviewed submitted version of manuscript: all authors. Approved the final version of the manuscript on behalf of all authors: Kikuchi. Study supervision: Kikuchi, Uesugi.

\section{Correspondence}

Naoya Kikuchi: Ibaraki Seinan Medical Center Hospital, Ibaraki, Japan.nhskikuchi@gmail.com. 